\title{
Ajustes Curriculares de las Escuelas de Odontología Chilenas en Periodo de Pandemia COVID-19
}

\author{
Curricular Adjustments of Chilean Dental Schools During the COVID-19 Pandemic Period
}

\author{
Daniel Flores-Repetto1; Camilo Pino'; Alejandra Letelier² \& Scarlette Hernández-Vigueras²
}

FLORES-REPETTO, D.; PINO, C.; LETELIER, A. \& HERNÁNDEZ-VIGUERAS, S. Ajustes curriculares de las escuelas de odontología chilenas en periodo de pandemia COVID-19. Int. J. Odontostomat. 15(4):857-866, 2021.

RESUMEN: Dada la expansión de la pandemia por COVID-19, se han implementado diversas medidas restrictivas, las cuales han afectado todos los ámbitos, incluido el educacional. A nivel internacional, las universidades han realizado diversos ajustes a sus programas curriculares para adaptarse a esta situación, siendo la carrera de Odontología particularmente afectada a causa de los cierres de clínicas y retraso en la adquisición de competencias clínicas. El objetivo del estudio es describir los principales ajustes curriculares de las carreras de Odontología de universidades chilenas, realizadas en periodo de pandemia en el año 2020, recopiladas a través de una encuesta. Se realizó un estudio observacional descriptivo, a través de una encuesta enviada a todos los representantes de las escuelas de Odontología de universidades chilenas. Se recopiló información sobre el contexto sanitario local, accesibilidad a las clínicas odontológicas, disponibilidad de tratamientos, vía de enseñanza, apoyo al bienestar mental e implicaciones futuras en la educación dental. De las 27 escuelas de Odontología nacionales, se obtuvieron 23 respuestas. Un 57\% de las escuelas tuvo limitaciones en el acceso y en el tipo de tratamientos odontológicos realizados en sus centros clínicos. Todas las escuelas implementaron una modalidad online para reemplazar las actividades lectivas presenciales y un $42 \%$ realizó actividades clínicas presenciales durante el año 2020. Todas las evaluaciones teóricas fueron realizadas de forma online, y un $82 \%$ de las evaluaciones de competencias clínicas se retrasaron para ser realizadas posteriormente. Debido a las medidas restrictivas de la pandemia, las clases presenciales, evaluaciones teóricas y algunas actividades prácticas se realizaron en modalidad online. Dependiendo del contexto comunal y políticas internas universitarias, algunas escuelas de odontología pudieron realizar actividades prácticas presenciales. El plan de estudios académico en las escuelas de odontología se ha visto afectado de diferentes maneras como resultado de la pandemia de COVID-19.

PALABRAS CLAVE: educación dental, COVID-1c9, encuesta, escuela dental, Curriculum vitae.

\section{INTRODUCCIÓN}

A finales de diciembre del año 2019 , se reportó un brote de neumonía de origen desconocido en la ciudad de Wuhan, China (Ludwig \& Zarbock, 2020). A inicio de enero de 2020 , se anunció que el virus correspondía a una nueva cepa de Coronavirus, denominándose posteriormente SARS-CoV-2 (Pingzheng et al., 2020) y se definió como el agente causal de la enfermedad de Coronavirus 2019 (COVID-19), nombre oficial dado por la Organización Mundial de la Salud (OMS) (World Health Organization, 2020a). El 11 de marzo de 2020, la
OMS declaró estatus de pandemia, dada la expansión global del virus (World Health Organization, 2020b).

Los gobiernos han tenido que adoptar medidas sanitarias excepcionales para frenar el avance de dicha enfermedad. Entre las recomendaciones que realizó la OMS para detener la propagación del virus, se encuentran el distanciamiento físico, uso de mascarilla y lavado de manos frecuente (World Health Organization, 2020c).

${ }^{1}$ Escuela de Odontología, Facultad de Medicina, Universidad Austral de Chile, Valdivia, Chile

2 Instituto de Odontoestomatología, Facultad de Medicina, Universidad Austral de Chile, Valdivia, Chile.

Esta investigación está basada en parte de los requerimientos de Daniel Flores Repetto y Camilo Pino Díaz para su titulación de Cirujano Dentista en la Escuela de Odontología de la Universidad Austral de Chile.

Received : 2021-07-02 Accepted: 2021-09-07 
En Chile, el estado de excepción constitucional de catástrofe fue declarado el 18 de marzo de 2020 (Diario Oficial de la República de Chile, 2020). Las medidas de prevención adoptadas han ido evolucionando, a medida que la OMS daba más información respecto del COVID-19, hasta que se desarrolló a nivel nacional un plan sanitario llamado "Paso a Paso", el cual trata de 5 pasos graduales, que varía según la situación sanitaria de cada comuna en particular, y abarca desde el paso 1, el cual implica una cuarentena total con permisos reducidos para realizar solo actividades esenciales, hasta el paso 5, en donde, no se aplica la cuarentena y los locales funcionan con un $75 \%$ de su capacidad máxima (Gobierno de Chile, 2020). En consecuencia, la cotidianidad se ha visto fuertemente afectada, abarcando áreas tales como la actividad laboral, las relaciones sociales, la educación, entre otras.

En el ámbito de educación superior, a partir del 16 de marzo del año 2020, las universidades chilenas tuvieron que suspender sus clases lectivas presenciales (Consejo de Rectores de las Universidades Chilenas, 2020), viéndose forzadas a realizar diferentes ajustes curriculares, con el fin de continuar impartiendo las actividades académicas. En el caso de la carrera de Odontología, los ajustes curriculares son especialmente complejos ya que la malla curricular contempla un alto porcentaje de actividades prácticas presenciales de simulación y clínicas. Los ajustes curriculares son definidos como un tipo de estrategia educativa, que puede implementarse para todos los estudiantes. Consiste en la adecuación del currículum de un determinado nivel educativo, con el fin de hacer que objetivos o contenidos en concreto sean más accesibles a un estudiante (Baumgart et al., 1982). Se describen dos tipos de ajustes curriculares, significativos y no significativos. El primero hace referencia cuando un estudiante no es capaz de alcanzar los objetivos principales del plan de estudios, teniendo que modificarse aspectos básicos del currículum. Por otro lado, el ajuste curricular no significativo corresponde a adaptaciones en cuanto a los tiempos, el ambiente, las actividades, la metodología, o manera de realizar la evaluación. Pueden suponer pequeñas variaciones en los contenidos, pero sin implicar un desfase curricular de más de un año académico. Este último, es el que fue utilizado por las distintas instituciones, producto de la pandemia en el año 2020 (Fundación CADAH, 2013).
A nivel internacional, existe poca información acerca de los ajustes curriculares de las carreras de Odontología durante el periodo de pandemia COVID19. La Asociación Europea de Educación Dental (ADEE), realizó en marzo del año 2020 un estudio descriptivo utilizando una encuesta, como herramienta de recolección de datos (Quinn et al., 2020). Además, la Asociación de Educación Dental del Asia Pacifico (ADEAP) organizó 3 simposios, en los que participaron 19 universidades de distintos países, de manera online (Chang et al., 2021). En Latinoamérica, en un reciente estudio brasileño se recopiló a través de una encuesta la información de 230 programas (Sponchiado-Junior et al., 2021). De estos 3 estudios, se puede observar que las actividades lectivas presenciales fueron suspendidas producto de la pandemia y se implementó una modalidad online para el reemplazo de éstas, por lo que, las actividades teóricas, las evaluaciones y parte de las actividades prácticas se realizaron de manera virtual. En las clínicas odontológicas que permanecieron abiertas, se realizó solamente tratamiento de urgencia y emergencia dental por el personal académico. Los estudiantes no podían realizar tratamientos odontológicos, a excepción de Taiwán, el cual, hasta el 10 de junio del 2020, mantuvo sus actividades tanto clínicas como teóricas de manera normal (Chang et al.).

En Chile, todavía no existen estudios acerca de los ajustes curriculares que se han hecho en las diversas escuelas de Odontología durante el periodo de pandemia. Es importante investigar estos cambios porque nos permite obtener un registro global sobre los ajustes curriculares que se realizaron durante el año 2020 en las Escuelas de Odontología de Chile. De esta forma, podremos conocer el modo en que se vieron afectadas las generaciones que cursaron durante dicho año, y la forma en que podría impactar en el futuro. Además, poder evaluar qué resultados traerá consigo la implementación drástica de esta nueva modalidad, y comparar los ajustes curriculares y cambios realizados en las instituciones chilenas con lo realizado en otras universidades extranjeras, lo cual, nos ayudaría a obtener una mejor perspectiva sobre nuestra realidad.

Por lo tanto, el principal objetivo de este estudio es describir los principales ajustes curriculares de las carreras de Odontología de universidades chilenas, realizados en periodo de pandemia en el año 2020 , recopilados a través de una encuesta. 


\section{MATERIAL Y MÉTODO}

Se realizó un estudio observacional de tipo descriptivo. La información se recopiló a través de una encuesta enviada a las autoridades de todas las escuelas de Odontología Chilenas. El presente estudio cuenta con la aprobación del Comité Ético Científico del Servicio de Salud Valdivia Ord. N. 412. La cantidad total de universidades que poseen escuelas de Odontología en Chile fue buscada a través del sitio web del Consejo Nacional de Educación de Chile (Consejo Nacional de Educación de Chile, 2021). La página web de cada universidad fue buscada a través del buscador "Google" y posteriormente se indagó el nombre de cada autoridad de las escuelas de Odontología, con sus respectivos e-mails de contacto.

Se definió el siguiente criterio de inclusión: Universidades chilenas que imparten la carrera de Odontología.

La encuesta utilizada está basada en la encuesta creada por la ADEE y socios de O-HealthEdu Erasmus plus, la cual consta de 13 preguntas de selección múltiple y se utilizó para el estudio de título "Thelmmediate Response of European Academic Dental Institutions and Future Implications for Dental Education" (Quinn et al.). Dicha encuesta fue traducida el español y adaptada al contexto chileno.

La encuesta traducida consta de un total de 14 preguntas, siendo 13 cerradas y 1 pregunta abierta. Se envió previamente a 10 docentes pertenecientes al Instituto de Odontoestomatología de la Universidad Austral de Chile, como método de validación de la traducción de la encuesta.

La encuesta se realizó a través de un formulario de Google, cuyo link fue enviado por correo electrónico a cada una de las autoridades. Al abrir el link, se presentaba el estudio junto a un consentimiento informado, el cual, en caso de aceptar, se desplegaba la encuesta.

Los datos fueron recopilados entre el 29 de marzo al 4 de mayo del 2021, a través de una hoja de cálculo de Google.

Para el análisis de los datos se utilizó estadística descriptiva, obteniendo frecuencias y porcentajes para las variables cualitativas de naturaleza nominal y ordinal. La estadística se realizó con el software estadístico SPSS versión 25 y Microsoft Excel.

\section{Encuesta Ajustes curriculares Odontología:}

* Indique su correo electrónico:

* ¿A cuál Universidad usted pertenece?

*¿Cuál es su cargo dentro de la carrera?

Instrucciones: Responda las preguntas según la alternativa que más predominó en el año 2020.

1. ¿A partir de qué fecha se suspendieron las actividades presenciales?

2. El contexto sanitario actual del lugar donde se ubica la clínica odontológica universitaria es: (El número de la respuesta está en relación al plan sanitario "Paso a Paso")

a) 1. Cuarentena Total

b) 2. Transición - Cuarentena solo fin de semana

c) 3. Preparación - Levantamiento de cuarentena, excepto grupo de riesgo.

d) 4. Apertura Inicial - Se puede retomar actividades de menor riesgo sin aglomeración.

e) 5. Apertura Avanzada - Permite aumentar la cantidad de gente en lugares determinados.

3. En algún periodo entre Marzo-Diciembre del año 2020, la comuna donde se encuentra ubicada la clínica odontológica universitaria estuvo en cuarentena total? (Paso 1)

a) Sí

b) No

4. La clínica odontológica de su universidad se encontró:

a) Abierta, acceso para estudiantes de pregrado, postgrado, académicos y funcionarios.

b) Abierta, pero con acceso solo a estudiantes de postgrados, académicos y funcionarios.

c) Abierta, pero con acceso solo a académicos y funcionarios.

d)Cerrado, sin acceso para académicos, in-vestigadores, estudiantes y funcionarios.

5. ¿Cuáles fueron las actividades permitidas en las clínicas odontológicas de su universidad? (Seleccione todas las que aplique, en caso de que la respuesta no se encuentre en las alternativas, especifique en Otros)

a) Disponibilidad de todo tipo de tratamiento.

b) Tratamientos/procedimientos sin generación de aerosoles.

c) Solo tratamientos de emergencia dental. (Ejemplos: Hemorragia, sangrado profuso, Trauma maxilofacial con compromiso vías aéreas)

d) Urgencia, tratamientos dentales impostergables. (Ejemplos: Pulpitis, pericoronaritis, TDA, complicaciones post -exodoncias)

e) Tratamientos dentales para pacientes vulnerables. (motivos de salud, edad, necesidades especiales)

f) No hay tratamientos disponibles, clínicas cerradas.

g) Otros: 
6. Si estuvo abierta la clínica odontológica, ¿a quién se le permitió asistir y qué actividad pudo realizar? (Seleccione todas las que aplique) (Si la clínica está cerrada, omita esta pregunta)

a) Estudiantes pregrado (actividad clínica)

b) Estudiantes pregrado (actividades de simulación)

c) Estudiantes postgrado (actividad clínica)

d) Estudiantes postgrado (actividades de simulación)

e) Personal académico para actividades clínicas.

7. ¿Qué factores determinaron cómo la clínica universitaria estuvo respondiendo ante la crisis del Covid-19? (Seleccione todas las que aplique)

a) Literatura científica o guías clínicas internacionales

b) Protocolos propios estandarizados establecidos para enfermedades infecciosas.

c) Pautas o directrices nacionales.

8. Si el acceso a la escuela dental estuvo limitado, ¿la universidad implementó educación virtual para reemplazar las actividades lectivas? (En caso de que su respuesta sea afirmativa, especifique la(s) plataforma(s) más utilizada(s) en Otros:

a) Sí

b) No

c) Otros:

9. Si el acceso a la escuela dental estuvo limitado, ¿Cómo se llevaron a cabo las evaluaciones? (Seleccione todas las que aplique) (Si la respuesta no se encuentra en las alternativas, especifique en Otros)

a) Todas las evaluaciones tanto teóricas como prácticas fueron pospuestas.

b) Evaluaciones teóricas fueron realizadas en modalidad online.

c) Evaluaciones prácticas fueron realizadas en modalidad online.

d) Algunas evaluaciones se realizaron de forma presencial.

e) Algunas evaluaciones se pospusieron para ser realizadas de manera presencial.

f) Otros:

10-Si el acceso a la escuela dental estuvo limitado, ¿pospusieron la evaluación de competencias clínicas adquiridas por los estudiantes?

a) Sí

b) No

11. ¿Se brindó apoyo específico al personal académico que sufrió estrés debido a la pandemia? (Seleccione todas las que aplique)

a) No hubo nuevo apoyo disponible

b) Sí, existió apoyo a nivel universitario central.

c) Sí, existió apoyo a nivel de Facultad/Escuela dental

Por favor responda el grado de acuerdo o desacuerdo de los siguientes enunciados:

12. La crisis del Covid-19 cambiará el futuro de la educación dental: a) Muy en acuerdo

b) Acuerdo

c) Desacuerdo

d) Muy en desacuerdo

13. La crisis del Covid-19 cambiará la forma en que se desempeñan el equipo de salud dental.

a) Muy en acuerdo

b) Acuerdo

c) Desacuerdo

d) Muy en desacuerdo

14. La educación en salud se modificará por la crisis del Covid-19:

a) Muy en acuerdo

b) Acuerdo

c) Desacuerdo

d) Muy en desacuerdo

\section{RESULTADOS}

Se encontró un total de 27 escuelas de Odontología, correspondiente a 19 universidades a nivel nacional. Se obtuvo la información de contacto de cada autoridad en las respectivas páginas web oficiales de las universidades. Tras enviar la encuesta a través de correo electrónico, se recopiló un total de 23 respuestas de 18 universidades nacionales, correspondiendo a una tasa de respuesta del $85 \%$ del total de escuelas de Odontología, y del $95 \%$ del total de universidades que imparten la carrera de Odontología.

Los resultados se encuentran ordenados en 5 áreas: i. Contexto sanitario local, ii. Accesibilidad y disponibilidad de tratamiento, iii. Vía de enseñanza y de evaluaciones, iv. Apoyo al bienestar mental al personal académico, v. Implicancias futuras en la educación dental.

Respecto al contexto sanitario local, las escuelas reportaron que de acuerdo con el plan sanitario: "Paso a Paso", el paso predominante en las distintas comunas donde se emplazan las escuelas de Odontología durante el año 2020 fue el Paso 1 (Cuarentena Total) con un $87 \%$ de respuesta, el resto indicó ser el Paso 2 (Transición). En el mismo sentido, un $100 \%$ de las escuelas indicó que la comuna donde se emplaza la clínica odontológica estuvo en el Paso 1 (Cuarentena Total) en algún momento del año 2020. Producto del inicio de la pandemia en el país, las distintas universidades debieron suspender sus actividades lectivas presenciales a principio de año, 
las fechas de suspensión de las 23 escuelas se observan en la Tabla I, en donde, un $52 \%$ de las escuelas suspendieron sus actividades a partir del 16 de marzo del 2020.

Tabla I. Fechas de suspensión de las actividades lectivas presenciales en el año 2020.

\begin{tabular}{lll}
\hline Dia y Mes & Frecuencia & Porcentaje (\%) \\
\hline 9 de marzo & 1 & 4.35 \\
16 de marzo & 12 & 52.2 \\
17 de marzo & 1 & 4.35 \\
18 de marzo & 3 & 13 \\
20 de marzo & 2 & 8.7 \\
23 de marzo & 2 & 8.7 \\
30 de marzo & 1 & 4.35 \\
1 de mayo & 1 & 4.35 \\
\hline
\end{tabular}

En relación a la accesibilidad a las clínicas odontológicas de las diferentes escuelas, los resultados se pueden observar en la Tabla II. Los datos de los tratamientos odontológicos disponibles, en las clínicas universitarias que estuvieron abiertas, se observan en la Tabla III.

Respecto a las actividades clínicas, en el caso de las escuelas que pudieron tener actividades presenciales, éstas fueron realizadas por el personal académico $(100 \%)$, un $70 \%$ de los estudiantes de postgrado y en menor medida, participaron estudiantes de pregrado (58\%). En las clínicas que se mantuvieron abiertas, en un $35,2 \%$ realizaron actividades de simulación, de las cuales, en un $11.7 \%$ asistieron solamente estudiantes de pregrado, y en un $23.5 \%$ podían asistir tanto estudiantes de pregrado como de postgrado.
En cuanto a las vías de enseñanza y modalidad de evaluación, se obtuvo que un $100 \%$ de las universidades implementó educación online para reemplazar las actividades lectivas presenciales, dentro de esta totalidad, un $58 \%$ de las universidades implementó únicamente educación online, mientras que un $42 \%$ realizó una enseñanza mixta, es decir, educación online y actividades presenciales, a través de actividades clínicas y/o de simulación; ninguna de las escuelas encuestadas realizó una enseñanza netamente presencial.

Respecto a la modalidad de evaluación, un 82 $\%$ de las evaluaciones de competencias clínicas se retrasaron para ser realizadas posteriormente, $100 \%$ de las escuelas indica que las evaluaciones teóricas se realizaron de manera virtual. En cuanto a la modalidad de evaluaciones prácticas, un $58 \%$ de estas evaluaciones fueron pospuestas para ser realizadas de manera presencial, un $33 \%$ se realizó presencialmente durante el año 2020 y un $9 \%$ fue realizado de forma online.

Se reporta que las clínicas universitarias usaron como base para sus protocolos distintos textos protocolizados para poder responder ante esta pandemia, obteniendo que un $87 \%$ usó literatura científica o guías clínicas internacionales, un $91 \%$ protocolos propios estandarizados establecidos para enfermedades infecciosas y un $96 \%$ pautas o directrices nacionales.

En relación a las ayudas en bienestar y manejo del estrés del personal académico, las escuelas de Odontología presentaron diferentes situaciones (Tabla

Tabla II. Accesibilidad a las clínicas odontológicas durante el periodo de pandemia del año 2020.

\begin{tabular}{lcc}
\hline \multicolumn{1}{c}{ Accesibilidad } & Frecuencia & $(\%)$ \\
\hline Abierta, acceso para estudiantes de pregrado, postgrado, académicos y funcionarios. & 10 & 43,5 \\
Abierta, pero con acceso solo a estudiantes de postgrados, académicos y funcionarios. & 3 & 13 \\
Abierta, pero con acceso solo a académicos y funcionarios. & 4 & 17.4 \\
Cerrado, sin acceso para académicos, investigadores, estudiantes y funcionarios. & 6 & 26,1 \\
*Estos datos fueron recopilados según la situación predominante del periodo de pandemia año 2020. &
\end{tabular}

Tabla III. Actividades permitidas en las clínicas odontológicas.

\begin{tabular}{lcc}
\multicolumn{1}{c}{ Disponibilidad de tratamiento } & Frecuencia & Porcentaje (\%) \\
\hline Todo tipo de tratamiento & 8 & 34,8 \\
Tratamientos/procedimientos sin generación de aerosoles & 6 & 26,1 \\
Solo tratamientos de emergencia dental & 9 & 39,1 \\
Urgencia, tratamientos dentales impostergables & 15 & 65,2 \\
Tratamientos dentales para pacientes vulnerables & 6 & 30,4 \\
No hay tratamientos disponibles, clínicas cerradas & 6 & 26,1 \\
\hline
\end{tabular}

*Las autoridades podían seleccionar más de una alternativa en esta pregunta. 
IV), donde en un $69 \%$, las ayudas se brindaron exclusivamente de manera centralizada por las universidades.

Tabla IV. Apoyo al personal académico frente al estrés.

\begin{tabular}{lcc}
\hline \multicolumn{1}{c}{ Apoyo a Salud Mental } & Frecuencia & $(\%)$ \\
\hline No hubo nuevo apoyo disponible & 1 & 4,3 \\
$\begin{array}{l}\text { Sí, existió apoyo a nivel } \\
\text { universitario central }\end{array}$ & 16 & 69,6 \\
$\begin{array}{l}\text { Sí, existió apoyo a nivel de } \\
\text { Facultad/Escuela dental }\end{array}$ & 4 & 17,4 \\
Sí, existió apoyo de ambos niveles & 2 & 8,7 \\
\hline
\end{tabular}

Finalmente, respecto a la opinión de las autoridades universitarias sobre 3 enunciados de implicancias futuras, se obtuvo que un $100 \%$ de las autoridades están de acuerdo sobre que esta crisis cambiará el futuro de la educación, tanto en salud como odontológico, y un $95 \%$ está de acuerdo en que esta pandemia modificará la forma en que se desempeñan los equipos odontológicos de salud.

\section{DISCUSIÓN}

Los ajustes curriculares han sido variados, acorde a los distintos escenarios producidos por la pandemia en las comunas del país, lo que ha definido el impacto y las adecuaciones curriculares de las distintas escuelas de Odontología de Chile. Un 78\% de las escuelas indican que suspendieron sus actividades lectivas presenciales durante la tercera semana de marzo del año 2020, estando en consonancia con la declaración del estado de catástrofe de Chile producto de la pandemia COVID19. Además, si bien las fechas son similares a la suspensión de actividades presenciales que hubo en otros países de Europa, tales como: Italia, Inglaterra (Alzahrani et al., 2020), existió una amplia diferencia en el contexto sanitario en ese momento, puesto que los países europeos, ya se encontraban en una difícil situación sanitaria, mientras que, en Chile, estaban recién comenzando los primeros contagios.

La predominancia de la cuarentena total en las distintas comunas donde se emplazan las escuelas de Odontología puede explicarse, ya que el complejo contexto sanitario, llevó a que las autoridades de salud decidan mantener la cuarentena total en las principales ciudades del país, durante gran parte del año 2020.
En las escuelas de Odontología chilenas, un 74 $\%$ de las clínicas universitarias estuvieron abiertas, y en un $58 \%$, los estudiantes podían acceder y realizar actividades clínicas. Estos resultados difieren con lo reportado en Europa a principio del año 2020 (Quinn et al.) y en otras partes del mundo (Chang et al.), donde los estudiantes no podían acceder ni realizar actividades clínicas. En un estudio recientemente publicado, en la que se encuestó a escuelas de odontología brasileñas, se reporta que, en cuanto a la accesibilidad a las clínicas odontológicas, un $95.6 \%$ de los programas suspendió todo tipo de actividad práctica presencial y tan sólo un $5.4 \%$ de las clínicas estuvo abierta (Sponchiado-Junior et al.). Estas diferencias, pueden deberse principalmente a la fecha en que se recopiló la información, puesto que los estudios citados fueron realizados durante el primer semestre del año 2020, a diferencia de este estudio que abarca desde marzo hasta diciembre del 2020 en donde, con el transcurso de los meses, hubo un aumento en el conocimiento de la enfermedad sumado a la creación de protocolos y normas sanitarias para minimizar el riesgo de contagio, lo que pudo permitir el acceso de los estudiantes de pregrado y/o postgrado a las clínicas odontológicas universitarias, principalmente durante la segunda mitad del año 2020. En un estudio realizado en la Universidad de Kuwait (Baskaradoss \& Al-Asfour, 2021), se indica que, a partir de septiembre del año 2020, los estudiantes recién podían realizar tratamientos odontológicos que no fueran generadores de aerosol. A su vez, en el presente estudio se encontró que, de las 17 escuelas que realizaron tratamientos odontológicos, 6 declararon realizar tratamientos sin generación de aerosol.

En el mismo sentido, en las clínicas que estuvieron abiertas, se realizaron principalmente actividades de urgencia y emergencia dental, esto se explica ya que de esta forma se prioriza y atiende sólo a pacientes que necesiten atención impostergable en salud, manteniendo el menor flujo de pacientes posible. Estos resultados coinciden con lo reportado en Europa a principio de la pandemia (Quinn et al.), con lo observado en Brasil (Sponchiado-Junior et al.) y con lo recomendado por la Asociación Dental Americana (ADA) (ADA Center for Professional Success, 2020).

A nivel nacional, la accesibilidad de una escuela dependió del número del plan: "Paso a Paso" en que se encontrase la comuna respectiva, esto significó que, al mismo tiempo, podían existir escuelas abiertas a la atención de público y otras cerradas. En ciertos momentos del año 2020, ocurrió que escuelas estando 
en el mismo número del plan "Paso a Paso", tenían distinta accesibilidad a sus clínicas odontológicas, dicha diferencia pudo darse ya sea por políticas propias de cada universidad, como por ciertas medidas restrictivas otorgadas por algunas SEREMI Regionales. Situación que también se repitió con los tipos de tratamientos que se podían realizar (Colegio de Cirujano Dentistas de Chile, 2020).

En cuanto a las vías de enseñanza, esta se realizó un $58 \%$ solamente vía online y un $42 \%$ a través de modalidad mixta (educación online y práctica clínica presencial), lo que se explicaría por los motivos señalados anteriormente (políticas internas de cada universidad, situación del plan "Paso a Paso" comunal,entre otros). En el estudio de SponchiadoJunior et al., se detalla que, en Brasil, el $83.3 \%$ de todos los programas que continuaron la enseñanza teórica tras la suspensión de actividades presenciales, fueron realizadas en modalidad virtual, situación similar a la nacional (Sponchiado-Junior et al.).

Esta adaptación a una nueva modalidad online de manera drástica significó un aumento de gastos, tanto económico como recurso humano, ya sea por la compra de licencias de software, prestación de servicios tecnológicos a los estudiantes, capacitación al personal, entre otros. Al comparar estos datos con diversos estudios (Haridy et al., 2020; Quinn et al.;), el porcentaje de uso de plataformas virtuales es similar, lo cual, puede explicarse ya que actualmente gran parte de la población tiene acceso a internet y la modalidad online, es el mejor método para administrar conocimientos, sin generar contacto entre personas. Por otro lado, cada universidad decidió qué plataformas virtuales adquirir según sus necesidades para la emisión de clases, administración de documentos y realización de pruebas.

En relación a las actividades de simulación en las clínicas odontológicas, estas han continuado tanto en instituciones extranjeras como nacionales. A nivel nacional, estas actividades se realizaron en un $35 \%$ de las escuelas de Odontología, pudiendo asistir, dependiendo del caso, estudiantes de pregrado y postgrado. La herramienta de simulación es considerada como un método favorable para el desarrollo de habilidades psicomotrices y competencias clínicas (Nishioka et al., 2021). Por otro lado, la simulación en laboratorio es considerada uno de los métodos más seguros para que los estudiantes desarrollen habilidades psicomotrices (Baskaradoss \& Al-Asfour).
Respecto a la modalidad de evaluación, todas las escuelas indican que realizaron evaluaciones teóricas de manera online, independientemente si estas hayan tenido actividades de manera presencial o no. Un $82 \%$ de las escuelas pospuso o retardo la evaluación de competencias clínicas, en comparación a un $72 \%$ de lo reportado por el estudio de Quinn et al. Además, en el estudio de Haridy et al. reportaron que las evaluaciones formativas y sumativas en línea han sido la estrategia alternativa de elección adoptada por la mayoría de las escuelas de Odontología de todo el mundo, situación que se repite en Chile (Haridy et al.; Quinn et al.).

Diversos estudios internacionales, indican que tanto profesores como estudiantes, presentan una adecuada satisfacción, con la educación virtual implementada, la cual incluso podría mantenerse tras el término de la pandemia (Huynh, 2017; Bennardo et al., 2020; Schlenz et al., 2020; Goob et al., 2021). Sin embargo, estos resultados difieren con lo reportado por otros estudios, en donde, se indica que los estudiantes aceptan parcialmente esta nueva modalidad, ya que tenían problemas de conexión, falta de tecnologías, fatiga del aprendizaje virtual, entre otros (Hattar et al., 2021; losif et al., 2021). Esta diferencia podría explicarse por las diferentes realidades que existen en los distintos países del mundo, en donde, la falta de recursos puede ser un factor determinante a la hora de implementar adecuadamente esta nueva modalidad. En Chile, a nivel de las escuelas de Odontología, la información acerca de la satisfacción de los y las estudiantes, en el período de pandemia, aún no está disponible.

Las distintas instituciones universitarias, han tenido que implementar apoyo a nivel de bienestar mental al personal académico, puesto que, la pandemia ha generado un aumento en el estrés y ansiedad en la población debido a la duración de las cuarentenas, sobrecarga académica, miedo a la infección, frustración, aburrimiento e incertidumbre por falta de información (Gualano et al., 2020). En cuanto al manejo del estrés en el personal académico producido por la pandemia, los resultados de este estudio difieren con lo reportado en el estudio de Quinn et al., donde, en un $30 \%$ de las instituciones no se brindó apoyo al personal académico. Esta diferencia entre los resultados puede explicarse por el periodo de tiempo de pandemia que abarcó cada estudio, puesto que el de Quinn et al. abarcó solamente los primeros meses de pandemia, en donde, posiblemente no todas las instituciones hayan alcanzado a hacer 
los ajustes o implementar los apoyos necesarios. En cambio, este estudio abarcó todo el periodo de pandemia del año 2020, lo que aumenta la posibilidad de que las instituciones hayan implementado ayudas para el tópico de bienestar y salud mental. En un estudio realizado en Arabia Saudita, se determinó que estudiantes o familiares de pacientes contagiados con COVID-19, causaban un efecto negativo en el bienestar mental de los estudiantes, lo cual trae como consecuencia, que estos perciban que poseen peores habilidades psicomotrices. Además, se ha reportado que las medidas de control de infecciones en las clínicas odontológicas también tienen un efecto en el bienestar mental de los estudiantes (Alrashed et al., 2021).

Todas las autoridades universitarias consultadas, están de acuerdo sobre los enunciados de las implicaciones futuras. Esto quiere decir, que las autoridades ven y analizan los ajustes curriculares como una oportunidad para mejorar en la educación dental y en salud, ya que muchos cambios pueden llegar a ser beneficiosos y mantenerse tras el término de la pandemia. En un estudio se reporta que este modelo de aprendizaje virtual tiene el potencial de continuar, incluso cuando las instituciones ya no se vean afectadas por la pandemia del COVID-19 (Quinn et al.; Kennedy et al., 2021).

Con los resultados obtenidos en este estudio, podemos obtener un registro sobre los ajustes curriculares y cambios que se realizaron durante el año 2020 en las Escuelas de Odontología de Chile, y el modo en que se vieron afectadas las generaciones que cursaron durante dicho año. Además, con ello podremos comparar y evaluar en el futuro las consecuencias o beneficios sobre la implementación drástica de esta nueva modalidad. Por otro lado, nos permite comparar con los ajustes curriculares y cambios realizados en universidades extranjeras, lo cual, nos brinda una mejor perspectiva sobre nuestra realidad y poder adoptar de manera permanente algún ajuste o cambio que sea beneficioso.

Dentro de las fortalezas del estudio, se encuentra la alta representatividad obtenida de las escuelas de Odontología chilenas. Además, se pudo abarcar ajustes curriculares realizados tanto en Universidades Estatales, Universidades Privadas del Consejo de Rectores de las Universidades Chilenas ( $\mathrm{CRUCH})$ y Universidades Privadas No Pertenecientes al $\mathrm{CRUCH}$, lo que da cuenta del panorama general de las escuelas de Odontología a nivel país.
Dentro de las limitaciones de este estudio, se abordó de manera general algunos tópicos, sin hacer énfasis o detallar cómo y qué evaluaciones y actividades prácticas online se realizaron específicamente. Otra limitación es que se consultó por resultados predominantes durante el año 2020 , por lo que cambios menores que pudieron haber sido significativos, puede que hayan pasado desapercibidos.

Se sugiere realizar estudios similares en los años siguientes, puesto que no se sabe cuánto tiempo más durará la pandemia y qué cambios pueden ser necesarios los próximos años, antes de volver a una "normalidad". Además, realizar estudios en el futuro para evaluar los efectos y la realidad de las generaciones que fueron educadas con estas modalidades, con el fin de analizar la presencia de fortalezas o debilidades, y acorde a esto, establecer la necesidad de crear programas remediales para solventar dichas debilidades, en el caso que no se haya podido cumplir con el perfil de egreso que declaran las universidades para sus estudiantes.

Del estudio se concluye que los principales ajustes curriculares de las escuelas de Odontología, fueron la adecuación de sus actividades a una modalidad netamente online en un poco más de la mitad de los encuestados, seguido por la modalidad mixta. Los ajustes curriculares se mantendrán en su mayoría, hasta retomar una nueva "normalidad" e incluso una vez iniciada ésta. Estudios futuros son necesarios para evaluar el impacto de la educación odontológica durante el periodo de pandemia.

\section{AGRADECIMIENTOS}

A Directores/as y Decanos/Decanas de las universidades chilenas que imparten la carrera de Odontología por la colaboración para este estudio.

Se agradece al Comité Ético Científico Servicio de Salud Valdivia por el oficio de la aprobación del estudio.

A la Asociación Chilena de Enseñanza a la Odontología. (ACHEO)

FLORES-REPETTO, D.; PINO, C.; LETELIER, A. \& HERNÁNDEZ-VIGUERAS, S. Curricular adjustments of Chilean dental schools during the COVID-19 pandemic period. Int. J. Odontostomat., 15(4):857-866, 2021. 
ABSTRACT: The COVID-19 pandemic has caused several disruptions in medical education worldwide. Universities made curricular adjustments in order to adapt to this situation. Dentistry has been particularly affected by the lockdown measures resulting in the closure of dental clinics and the disruption of face to face personal training of students. The objective of this study was to describe the main curriculum adjustments made by the dental schools in Chile during 2020. A cross-sectional online survey was carried out which included representatives from all Dental Schools in Chilean Universities. The survey requested information related to local sanitary situations, accessibility to dental clinics, availability of treatments, teaching modality, support for mental well-being and future implications of dental education. Of 27 dental schools a total of 23 responses were received. It was found that $57 \%$ of schools had limited access to dental clinics. All schools had implemented online modality to replace face-to-face theoretical training, $42 \%$ were able to conduct clinical activities during 2020 and $82 \%$ of clinical competency assessments were postponed. Due to the restrictive measures of the pandemic, the face-to-face classes, theoretical evaluations and some practical activities were performed online. Depending on the communal context, dental schools were able to execute practical activities. The academic curriculum in dental schools has been severely affected in different ways as a result of the COVID-19 pandemic.

KEY WORDS: dental education, COVID-19, survey, dental school, curriculum.

\section{REFERENCIAS BIBLIOGRÁFICAS}

ADA Center for Professional Success. COVID-19 State Mandates and Recommendations. United States, ADA Center for Professional Success, 2020. Disponible en:https:// success.ada.org/en/practice-management/patients/covid-19$\mathrm{s}$ t a t e - mand a te s-and-recommend a tions? utm_source=adaorg\&utm_medium $=$ covid-resourcesIp\&utm_content=stateaction\&utm_campaign $=$ covid$19 \&$ \& ga $=2.164286717 .333362180 .1586291400$ 2065192682.1579010925

Alrashed, F.; Sattar, K.; Habib, S.; Ahmad, T.; Al Rashoud, A.; Safhi, T.; Almajed, A.; Alnafisah, H.; Alharbi, N. \& Alsubiheen, A. Implications of the COVID-19 pandemic on the mental health and professional psychomotor skills of dental students. Psychol. Res. Behav. Manag., 14:675-85, 2021.

Alzahrani, S.; Alrusayes, A. \& Aldossary, M. Impact of COVID-19 pandemic on dental education, research, and students. Int. J. Health Sci. Res., 10(6):207-12, 2020.

Baskaradoss, J. \& Al-Asfour A. Dental education in an era of COVID19: kuwait's experience. Int. J. Environ. Res. Public. Health, 18(11):5606, 2021.

Baumgart, D.; Brown, L.; Pumpian, I.; Nisbet, J.; Ford, A.; Sweet, M.; Messina, R. \& Schroeder, J. Principle of partial participation and individualized adaptations in educational programs for severely handicapped students. J. A. S. H., 7(2):17-43, 1982.

Bennardo, F.; Buffone, C.; Fortunato, L. \& Giudice, A. COVID-19 is a challenge for dental education-A commentary. Eur. J. Dent. Educ., 24(4):822-4, 2020.
Chang, T.; Hong, G.; Paganelli, C.; Phantumvanit, P.; Chang, W.; Shieh, Y. \& Hsu, M. Innovation of dental education during COVID-19 pandemic. J. Dent. Sci., 16(1):15-20, 2021.

Colegio de Cirujano Dentistas de Chile. Comunidad odontológica de la Araucanía solicitó unida el fin de la prohibición de atenciones dentales. Santiago de Chile, Colegio de Cirujano Dentistas de Chile, 2020. Disponible en: http:// www.colegiodentistas.cl/inicio/2020/05/01/comunidadodontologica-de-la-araucania-solicito-unida-el-fin-de-laprohibicion-de-atenciones-dentales

Consejo de Rectores de las Universidades Chilenas ( $\mathrm{CRUCH})$. Universidades del consejo de rectores suspenden clases para prevenir contagio de coronavirus. Santiago de Chile, Consejo de Rectores de las Universidades Chilenas, 2020. Disponible en: https://www.consejoderectores.cl/noticia/865/ universidades-del-consejo-de-rectores-suspende n-clasespara-prevenir-contagio-de-coronavirus.html

Consejo Nacional de Educación de Chile. INDICES Base de Datos. Santiago de Chile, Consejo Nacional de Educación de Chile, 2021. Disponible en: https://www.cned.cl/bases-de-datos

Diario Oficial de la República de Chile. Publicación del Miércoles 18 de Marzo de 2020. Santiago de Chile, Diario Oficial de la República de Chile, 2020. Disponible en: https:// www.diariooficial.interior.gob.cl/publicaciones/2020/03/18/ 42607-B/01/1742691.pdf

Fundación Cantabría Ayuda al Déficit de Atención e Hiperactividad (Fundación CADAH). TDAH: Adaptaciones curriculares. Santander, España, Fundación Cantabría Ayuda al Déficit de Atención e Hiperactividad, 2013. Disponible en: https:// www.fundacioncadah.org/web/articulo/tdah-adaptacionescurriculares.html

Gobierno de Chile. Paso a Paso, Nos Cuidamos. Santiago de Chile, Gobierno de Chile, 2020. Disponible en: https:// www.gob.cl/pasoapaso/

Goob, J.; Erdelt, K.; Güth, J. \& Liebermann, A. Dental education during the pandemic: Cross-sectional evaluation of four different teaching concepts. J. Dent. Educ., 85(10):1574-87, 2021.

Gualano, M.; Lo Moro, G.; Voglino, G.; Bert, F. \& Siliquini, R. Effects of Covid-19 lockdown on mental health and sleep disturbances in Italy. Int. J. Environ. Res. Public Health, 17(13):4779, 2020.

Haridy, R.; Abdalla, M.; Kaisarly, D. \& El Gezawi, M. A crosssectional multi center survey on the future of dental education in the era of COVID-19: Alternatives and implications. J. Dent. Educ., 85(4):483-93, 2020.

Hattar, S.; AlHadidi, A.; Sawair, F.; Alraheam, I.; El-Ma'aita, A. \& Wahab, F. Impact of COVID-19 pandemic on dental education: online experience and practice expectations among dental students at the University of Jordan. BMC. Med. Educ., 21(1):151, 2021.

Huynh, R. The role of E-learning in medical education. Acad. Med., 92(4):430, 2017.

Iosif, L.; Tâncu, A.; Didilescu, A.; Imre, M.; Galbinau, B. \& Ilinca, R. Self-perceived impact of COVID-19 pandemic by dental students in bucharest. Int. J. Environ. Res. Public Health, 18(10):5249, 2021.

Kennedy, E.; White, B.; Weintraub, J.; Moss, M.; Jordan, S.; Quick, K.; Ticku, S.; Huang, B. \& Douglass, C. Collaborating and teaching a synchronous, multi-university, virtual course: Health policy and Access to dental care. J. Dent. Educ., 85(9):153642, 2021.

Ludwig, S. \& Zarbock, A. Corona viruses and SARS-CoV-2: A Brief Overview. Anesth. Analg., 131(1):93-6, 2020.

Nishioka, T.; Mayanagi, G.; Iwamatsu-Kobayashi, Y. \& Hong, G. Impact of the COVID-19 pandemic on dental clinical training and future prospects. J. Dent. Educ., 10.1002/jdd.12562, 2021. 
Pingzheng, M.; Yuanyuan, X.; Yu, X.; Liping, D.; Qiu, Z.; Hongling, W.; Yong, X.; Zhenshun, C.; Shicheng, G.; Ke, L.; Mingqi, L.; Tielong, C.; Shihui, S.; Zhiyong, M.; Xiaoping, C.; Ruiying, Z.; Qian, C.; Fan, W. \& Yongxi, Z. Clinical characteristics of refractory COVID-19 pneumonia in Wuhan, China. Clin. Infect. Dis., 2020.

Quinn, B.; Field, J.; Gorter, R.; Akota, I.; Manzanares, M.; Paganelli, C.; Davies, J.; Dixon, J.; Gabor, G.; Amaral Mendes, R.; Hahn, P.; Vital, S.; O'Brien, J.; Murphy, D. \& Tubert-Jeannin, S. COVID19: The immediate response of European academic dental institutions and future implications for dental education. Eur. J. Dent. Educ., 24(4):811-14, 2020.

Schlenz, M.; Schmidt, A.; Wöstmann, B.; Krämer, N. \& SchulzWeidner, N. Students' and lecturers' perspective on the implementation of online learning in dental education due to SARS-CoV-2 (COVID-19): a cross-sectional study. BMC. Med. Educ., 20:354, 2020.

Sponchiado-Júnior, E.; Vieira, W.; Silva, L.; Ferraz, C.; Affonso de Almeida, J.; Gomes, B. \& Soares, A. Impact of COVID-19 on dental education in Brazil. Rev. ABENO, 21(1):1225, 2021.

World Health Organization (WHO). Cronología de la respuesta de la OMS a la COVID-19. Ginebra, World Health Organization, 2020a. Disponible en: https://www.who.int/es/news/item/29-062020-covidtimeline

World Health Organization (WHO). Novel Coronavirus (2019-nCoV) SituationReport - 22. Ginebra, World Health Organization, 2020b. Disponible en: https://www.who.int/docs/default-source/ coronaviruse/situation-reports/20200211-sitrep-22ncov.pdf?sfvrsn=fb6d49b1 2

World Health Organization (WHO). Orientaciones para el público. Ginebra, World Health Organization, 2020c. Disponible en: https:/ /www.who.int/es/emergencies/diseases/novel-coronavirus-2019/ advice-for-public
Dirección para correspondencia:

Dra. Scarlette Hernández Vigueras Instituto de Odontoestomatología Rudloff \#1640

Valdivia

CHILE

E-mail: shernandez@uach.cl 